TRANSACTIONS OF THE

AMERICAN MATHEMATICAL SOCIETY

Volume 362, Number 7, July 2010, Pages 3365-3376

S 0002-9947(10)05102-0

Article electronically published on February 24, 2010

\title{
PSEUDO-REAL RIEMANN SURFACES AND CHIRAL REGULAR MAPS
}

\author{
EMILIO BUJALANCE, MARSTON D. E. CONDER, AND ANTONIO F. COSTA
}

\begin{abstract}
A Riemann surface is called pseudo-real if it admits anticonformal automorphisms but no anticonformal involution. In this paper, we study general properties of the automorphism groups of such surfaces and their uniformizing NEC groups. In particular, we prove that there exist pseudo-real Riemann surfaces of every possible genus $g \geq 2$. We also study pseudo-real surfaces of genus 2 and 3 . Further, we establish a connection between pseudoreal surfaces with maximal automorphism group, and chiral 3-valent regular maps, and use this to show there exist such surfaces for infinitely many genera, by exhibiting infinite families of chiral regular maps of type $\{3, k\}$ for all $k \geq 7$.
\end{abstract}

\section{INTRODUCTION}

A Riemann surface is called pseudo-real if it admits anticonformal automorphisms but no anticonformal involution.

Pseudo-real Riemann surfaces appear in a natural way in the study of the moduli space $\mathcal{M}_{g}^{K}$ of Riemann surfaces considered as Klein surfaces. If $\mathcal{M}_{g}$ is the moduli space of Riemann surfaces of genus $g$, then there is a twofold covering $\pi: \mathcal{M}_{g} \rightarrow$ $\mathcal{M}_{g}^{K}$. In other words, $\mathcal{M}_{g}^{K}$ can be considered as the orbit space of an involution $i$ on $\mathcal{M}_{g}$, sending each Riemann surface to its complex conjugate.

The fixed point set of $i$ is the preimage of the branched locus of $\pi$ and consists of all the Riemann surfaces of genus $g$ that are isomorphic to their conjugate. This set Fix $(i)$ contains, of course, the well-known (and extensively studied) real Riemann surfaces, which are those admitting an anticonformal involution — see [12, 15], for instance. But $\operatorname{Fix}(i)$ also contains surfaces that are not real surfaces, namely those which are equivalent to their conjugate but do not have any anticonformal involution. These are precisely the pseudo-real surfaces, which we study here.

Note that pseudo-real Riemann surfaces have non-trivial conformal automorphisms; hence the locus of pseudo-real Riemann surfaces in $\mathcal{M}_{g}$ is contained in the singular set of the orbifold $\mathcal{M}_{g}=\mathbb{T}_{g} / \operatorname{Mod}_{g}$.

In Section 3 we study general properties of the automorphism groups of these surfaces and the related uniformizing NEC groups. In particular, we prove that there are pseudo-real Riemann surfaces of every genus $g \geq 2$. In Section 4 we study pseudo-real surfaces of genus 2 and 3.

Received by the editors June 11, 2007.

2000 Mathematics Subject Classification. Primary 30F10; Secondary 14H10, 57M15.

The first author was partially supported by MTM2005-01637.

The second author was partially supported by N.Z. Marsden Fund UOA0721.

The third author was partially supported by MTM2005-01637.

(C)2010 American Mathematical Society Reverts to public domain 28 years from publication 
In recent years a vast literature has been published about real Riemann surfaces - that is, Riemann surfaces admitting anticonformal involutions - but very little is known about pseudo-real surfaces. A study of hyperelliptic pseudo-real Riemann surfaces was made in [18, and [2], and pseudo-real surfaces with cyclic automorphism group were investigated in [13.

In Section 5 we consider the maximal order of the automorphism group of a pseudo-real Riemann surface (relative to its genus), and we establish a connection between pseudo-real surfaces with maximal automorphism group, and chiral 3valent regular maps. Finally in Section 6 we show that there exist pseudo-real surfaces with automorphism group of maximal order for infinitely many genera, by proving the existence of concrete infinite families of chiral regular maps of type $\{3, k\}$ for $k \geq 7$.

\section{Preliminaries on Fuchsian and NEC groups}

A non-Euclidean crystallographic group (or NEC group) is a discrete group of isometries of the hyperbolic plane $\mathbb{D}$. We shall assume that an NEC group has a compact orbit space. If $\Gamma$ is such a group, then its algebraic structure is determined by its signature

$$
\left(h ; \pm ;\left[m_{1}, \ldots, m_{r}\right] ;\left\{\left(n_{11}, \ldots, n_{1 s_{1}}\right), \ldots,\left(n_{k 1}, \ldots, n_{k s_{k}}\right)\right\}\right) .
$$

The orbit space $\mathbb{D} / \Gamma$ is a surface, possibly with boundary. The number $h$ is called the genus of $\Gamma$ and equals the topological genus of $\mathbb{D} / \Gamma$, while $k$ is the number of its boundary components, and the sign is + or - according to whether or not the surface is orientable. The integers $m_{i} \geq 2$ are called the proper periods and represent the branched indices over interior points of $\mathbb{D} / \Gamma$ in the natural projection $\pi: \mathbb{D} \rightarrow \mathbb{D} / \Gamma$. The bracketed expressions $\left(n_{i 1}, \ldots, n_{i s_{i}}\right)$, some or all of which may be empty (with $s_{i}=0$ ), are called the period cycles and represent the branchings over the $i^{\text {th }}$ hole in the surface, and the numbers $n_{i j} \geq 2$ are the link periods.

Associated with each signature [5] there exists a canonical presentation for the group $\Gamma$, and a formula for the hyperbolic area of its fundamental domain. If the signature has sign + , then $\Gamma$ has the following generators:

$$
\begin{aligned}
& x_{1}, \ldots, x_{r} \text { (elliptic elements), } \\
& c_{10}, \ldots, c_{1 s_{1}}, \ldots, c_{k 0}, \ldots, c_{k s_{k}} \text { (reflections), } \\
& e_{1}, \ldots, e_{k} \text { (boundary transformations), } \\
& a_{1}, b_{1}, \ldots, a_{h}, b_{h} \text { (hyperbolic elements); }
\end{aligned}
$$

these generators satisfy the defining relations

$$
\begin{aligned}
& x_{i}^{m_{i}}=1 \quad(\text { for } 1 \leq i \leq r), \\
& c_{i j-1}^{2}=c_{i j}^{2}=\left(c_{i j-1} c_{i j}\right)^{n_{i j}}=1, c_{i s_{i}}=e_{i}^{-1} c_{i 0} e_{i} \quad\left(\text { for } 1 \leq i \leq k, 0 \leq j \leq s_{i}\right), \\
& x_{1} \ldots x_{r} e_{1} \ldots e_{k} a_{1} b_{1} a_{1}^{-1} b_{1}^{-1} \ldots a_{h} b_{h} a_{h}^{-1} b_{h}^{-1}=1 .
\end{aligned}
$$

If the sign is -, then we just replace the hyperbolic generators $a_{i}, b_{i}$ by glide reflections $d_{1}, \ldots, d_{h}$, and the last relation by $x_{1} \ldots x_{r} e_{1} \ldots e_{k} d_{1}^{2} \ldots d_{h}^{2}=1$.

The hyperbolic area of an arbitrary fundamental region of an NEC group $\Gamma$ with signature is given by

$$
\mu(\Gamma)=2 \pi\left(\varepsilon h-2+k+\sum_{i=1}^{r}\left(1-\frac{1}{m_{i}}\right)+\frac{1}{2} \sum_{i=1}^{k} \sum_{j=1}^{s_{i}}\left(1-\frac{1}{n_{i j}}\right)\right),
$$

where $\varepsilon=2$ if the sign is + , and $\varepsilon=1$ if the sign is -. Furthermore, any discrete group $\Lambda$ of isometries of $\mathbb{D}$ containing $\Lambda$ as a subgroup of finite index is also an 
NEC group, and the hyperbolic area of a fundamental region for $\Lambda$ is given by the Riemann-Hurwitz formula:

$$
[\Lambda: \Gamma]=\mu(\Gamma) / \mu(\Lambda) .
$$

For any NEC group $\Lambda$, let $\Lambda^{+}$denote the subgroup of orientation-preserving elements of $\Lambda$, called the canonical Fuchsian subgroup of $\Lambda$. If $\Lambda^{+} \neq \Lambda$, then $\Lambda^{+}$ has index 2 in $\Lambda$ and we say that $\Lambda$ is a proper NEC group.

Let $X$ be a compact Riemann surface of genus $g>1$. Then there is a Fuchsian surface group $\Gamma$ (that is, an NEC group with signature $(g ;+;[-] ;\{-\}))$ such that $X=\mathbb{D} / \Gamma$, and the automorphism group $\operatorname{Aut}(X)$ of $X$ is isomorphic to $\Delta / \Gamma$, where $\Delta$ is an NEC group containing $\Gamma$. We denote by $\operatorname{Aut}^{+}(X)$ the group $\Delta^{+} / \Gamma$ of all orientation-preserving automorphisms of $X$.

\section{Pseudo-real Riemann surfaces}

Definition 3.1. An anticonformal involution is an orientation-reversing automorphism of order 2. A Riemann surface is called pseudo-real if it admits anticonformal automorphisms but has no anticonformal involution.

Proposition 3.2. Let $X$ be a pseudo-real Riemann surface, and let $G$ be the group of conformal and anticonformal automorphisms of $X$. Then 4 divides the order of $G$.

Proof. Let $g$ be any anticonformal automorphism of $X$. If $g$ has order $2^{a} m$, where $m$ is odd, then $g^{m}$ is an anticonformal automorphism of order $2^{a}$, and then $a>1$ because $X$ is pseudo-real. Thus $G$ has an element $g^{m}$ of order divisible by 4 .

Theorem 3.3. Suppose the pseudo-real surface $X$ is conformally equivalent to $\mathbb{D} / \Gamma$, where $\Gamma$ is a surface Fuchsian group, and suppose $\Gamma$ is normalized by an $N E C$ group $\Delta$ such that $\Delta / \Gamma \cong G=\operatorname{Aut}(X)$. Then the signature of $\Delta$ has the form $\left(p ;-;\left[m_{1}, \ldots, m_{r}\right]\right)$, and if $\Delta^{+}$is the canonical Fuchsian subgroup of $\Delta$, then the signature of $\Delta^{+}$is

$$
\left(p-1 ;+;\left[m_{1}, m_{1}, m_{2}, m_{2}, \ldots, m_{r}, m_{r}\right]\right) .
$$

Proof. Since $G$ has anticonformal automorphisms, the signature of $\Delta$ is the signature of a proper NEC group, that is, a group with anticonformal transformations. Thus the signature of $\Delta$ has the form

$$
\left(p ;-;\left[m_{1}, \ldots, m_{r}\right]\right) \quad \text { or } \quad\left(p ; \pm ;\left[m_{1}, \ldots, m_{r}\right] ;\left\{\left(n_{i 1}, \ldots, n_{i k_{i}}\right)_{i=1, \ldots, l}\right\}\right) .
$$

Note that in the second case, $\Delta$ contains reflections. Now let us consider the monodromy epimorphism

$$
\theta: \Delta \rightarrow \Delta / \Gamma \cong G
$$

which sends anticonformal transformations to anticonformal automorphisms. If $\Delta$ contains reflections, that is, if $\Delta$ has signature

$$
\left(p ; \pm ;\left[m_{1}, \ldots, m_{r}\right] ;\left\{\left(n_{i 1}, \ldots, n_{i k_{i}}\right)_{i=1, \ldots, l}\right\}\right),
$$

where $l>0$, then the image by $\theta$ of a reflection is an anticonformal involution in $G$; hence the signature of $\Delta$ must be of the form $\left(p ;-;\left[m_{1}, \ldots, m_{r}\right]\right)$, with no boundary components. The signature of $\Delta^{+}$can now be obtained from the signature of $\Delta$ using the Riemann-Hurwitz formula and [5]. 
Theorem 3.4. For every integer $g \geq 2$, there exist pseudo-real surfaces of genus $g$.

Proof. Let $\Delta$ be an NEC group with signature $\left(\delta ;-;\left[2,{ }^{g+.}, 2\right]\right)$, where $\delta=\varepsilon=1$ if $g$ is even, or $\delta=2$ and $\varepsilon=-1$ if $g$ is odd. Let $x_{i}$ (for $1 \leq i \leq g+\varepsilon$ ) and $d_{j}$ (for $1 \leq j \leq \delta$ ) be a canonical system of generators of $\Delta$. We may define an epimorphism $\theta: \Delta \rightarrow \mathbb{Z}_{4}=\left\langle a: a^{4}=1\right\rangle$ by setting

$$
\theta\left(x_{i}\right)=a^{2} \text { for } 1 \leq i \leq g+\varepsilon, \quad \text { and } \theta\left(d_{j}\right)=a \text { for } 1 \leq j \leq \delta .
$$

Then $X=\mathbb{D} / \operatorname{ker} \theta$ is a Riemann surface such that $\Delta / \operatorname{ker} \theta \cong \mathbb{Z}_{4}$ is a group of automorphisms of $X$. Choosing $\Delta$ to be maximal (see [4]) ensures that the group $\Delta / \operatorname{ker} \theta$ will be the full automorphism group of $X$, and this will contain anticonformal automorphisms but only one involution, namely $a^{2}$, and that involution is conformal. Hence $X$ is pseudo-real.

\section{Pseudo-Real surfaces of Genus 2 AND 3}

Theorem 4.1. Let $X$ be a pseudo-real Riemann surface of genus 2. Then Aut $(X)$ is isomorphic to $\mathbb{Z}_{4}$, and if $A u t(X) \cong \Delta / \Gamma$, where $X=\mathbb{D} / \Gamma$, then $\Delta$ has signature $(1 ;-;[2,2,2])$.

Proof. If $G=\Delta / \Gamma$, then $G^{+}=\Delta^{+} / \Gamma$ is the conformal automorphism group of a Riemann surface of genus 2 . By Theorem 3 and [6, Table 4], we know that the only possibilities for $G^{+}$and the signature $s\left(\Delta^{+}\right)$of $\Delta^{+}$are the following:

$$
\begin{aligned}
G^{+} \cong \mathbb{Z}_{2} \text { and } s\left(\Delta^{+}\right)=(0 ;+;[2,2,2,2,2,2]), \\
\text { or } \quad G^{+} \cong \mathbb{Z}_{2} \text { and } s\left(\Delta^{+}\right)=(1 ;+;[2,2]), \\
\text { or } \quad G^{+} \cong \mathbb{Z}_{4} \text { and } s\left(\Delta^{+}\right)=(0 ;+;[2,2,4,4]) .
\end{aligned}
$$

The second and third of these three cases are ruled out by the analysis undertaken in [2], so we are left with only the first case. Then, since $\Delta$ must contain $\Delta^{+}$ as a subgroup of index two, the signature of $\Delta$ must be $(1 ;-;[2,2,2])$, and since $G^{+} \cong \mathbb{Z}_{2}$, also $G \cong \mathbb{Z}_{4}$.

We can easily construct such a pseudo-real Riemann surface $X$ of genus 2, for example, as in the proof of Theorem 3.4 (with $\delta=\varepsilon=1$ and $g=2$ ).

Theorem 4.2. Let $X$ be a pseudo-real Riemann surface of genus 3 , with automorphism group $G \cong \Delta / \Gamma$, where $X=\mathbb{D} / \Gamma$. Then there are three possible cases, all of which are realisable:

(a) $G \cong \mathbb{Z}_{4}$, and $\Delta$ has signature $(2 ;-;[2,2])$, or

(b) $G \cong D_{4}$, and $\Delta$ has signature $(1 ;-;[2,2,2])$, or

(c) $G \cong \mathbb{Z}_{4} \oplus \mathbb{Z}_{2}$, and $\Delta$ has signature $(1 ;-;[2,2,2])$.

Proof. The index 2 subgroup $G^{+} \cong \Delta^{+} / \Gamma$ of $G \cong \Delta / \Gamma$ is the automorphism group of a Riemann surface of genus 3. By Theorem 3 and [6, Table 4], and using the results of [2] and [3], we find that the only possibilities for $G^{+}$and the signature of $\Delta^{+}$are the following:

$$
\begin{aligned}
\quad G^{+} \cong \mathbb{Z}_{2} & \text { and } s\left(\Delta^{+}\right)=(0 ;+;[2,2,2,2,2,2,2,2]), \\
\text { or } \quad G^{+} \cong \mathbb{Z}_{2} & \text { and } s\left(\Delta^{+}\right)=(1 ;+;[2,2,2,2]), \\
\text { or } \quad G^{+} \cong \mathbb{Z}_{2} \oplus \mathbb{Z}_{2} & \text { and } s\left(\Delta^{+}\right)=(0 ;+;[2,2,2,2,2,2]) .
\end{aligned}
$$


In the first case, $G$ must be $\mathbb{Z}_{4}$ and $\Delta$ must have signature $(1 ;-;[2,2,2,2])$, but then there is no epimorphism $\theta: \Delta \rightarrow \mathbb{Z}_{4}$ with an appropriate kernel $\Gamma$, so this case is ruled out. From the other two cases, we deduce that the only possibilities for $G$, $G^{+}$and $\Delta$ are the following:

$$
\begin{aligned}
& G \cong \mathbb{Z}_{4}, \quad G^{+} \cong \mathbb{Z}_{2} \quad \text { and } \quad s(\Delta)=(2 ;-;[2,2]), \\
& \text { or } \quad G \cong D_{4}, \quad G^{+} \cong \mathbb{Z}_{2} \oplus \mathbb{Z}_{2} \quad \text { and } s(\Delta)=(1 ;-;[2,2,2]) \text {, } \\
& \text { or } \quad G \cong \mathbb{Z}_{4} \oplus \mathbb{Z}_{2}, \quad G^{+} \cong \mathbb{Z}_{2} \oplus \mathbb{Z}_{2} \quad \text { and } s(\Delta)=(1 ;-;[2,2,2]) \text {. }
\end{aligned}
$$

In each case it is easy to establish an epimorphism $\theta: \Delta \rightarrow G$ where $\Delta$ is a maximal NEC group with the corresponding signature, so that $G=\Delta / \operatorname{ker} \theta$ is the automorphism group of a pseudo-real Riemann surface of genus 3 .

\section{The MAXIMAL ORDER OF THE AUTOMORPHISM GROUP OF A PSEUDO-REAL RIEMANN SURFACE}

Theorem 5.1. If $X$ is a pseudo-real Riemann surface of genus $g$ with automorphism group $G$, then $|G| \leq 12(g-1)$. Moreover, if $|G|=12(g-1)$ and $G \cong \Delta / \Gamma$, where $X \simeq \mathbb{D} / \Gamma$, then the signature of $\Delta$ is $(1 ;-;[2,3])$.

Proof. By Theorem 3.3, the NEC group $\Delta$ has signature $\left(p ;-;\left[m_{1}, \ldots, m_{r}\right]\right)$, and then from the Riemann-Hurwitz formula, we find that

$$
2 g-2=|G|\left(p-2+\sum_{i=1}^{r}\left(1-\frac{1}{m_{i}}\right)\right) .
$$

The minimum positive value of the bracketed expression on the right-hand side is $\frac{1}{6}$, which is attained when $p=1, r=2, m_{1}=2$ and $m_{2}=3$, and in that case $|G|=12(g-1)$.

We are interested in the construction of pseudo-real Riemann surfaces with automorphism group of maximal order. In order to find such a pseudo-real surface of genus $g$ with maximal symmetry, we need to find:

(a) a maximal NEC group $\Delta$ with signature $(1 ;-;[2,3])$,

(b) a finite group $G$ of order $12(g-1)$, and

(c) an epimorphism $\theta: \Delta \rightarrow G$ such that $\operatorname{ker} \theta$ is a Fuchsian surface group of genus $g$.

Here we remark that the monodromy epimorphism $\theta$ is determined by the image of the canonical generators. If we have a group $G$ of order $12(g-1)$ and a monodromy epimorphism $\theta: \Delta \rightarrow G$, then the group $\Delta$ is maximal (see [16] and [5]) unless there is another NEC group $\Delta^{\prime}$ with signature $(0 ;+;[2],\{(2,3)\})$ containing $\Delta$ and an epimorphism $\theta^{\prime}: \Delta^{\prime} \rightarrow G^{\prime}$, where $G^{\prime}$ is an index two extension of $G$ and $\left.\theta^{\prime}\right|_{\Delta}=\theta$.

Proposition 5.2. Let $\Delta$ be an NEC group with signature $(1 ;-;[2,3])$, let $d, x_{1}$ and $x_{2}$ be the generators of a canonical presentation for $\Delta$, and let $\theta: \Delta \rightarrow G$ be an epimorphism such that $\theta\left(d_{1}\right)=a$ and $\theta\left(x_{1}\right)=b$. Then $\theta$ can be extended to an epimorphism $\theta^{\prime}: \Delta^{\prime} \rightarrow G^{\prime}$, where $\Delta^{\prime}$ is an NEC group containing $\Delta$ as a subgroup of index 2 and $G^{\prime}$ is a group containing $G$ as a subgroup of index 2 , if and only if $G$ admits an automorphism of order 2 such that $\alpha(a)=a^{-1}$ and $\alpha(b)=b^{-1}$. 
Proof. If $G$ admits such an automorphism $\alpha$, then we can construct the semidirect product $G^{\prime}=G \rtimes_{\alpha} \mathbb{Z}_{2}$, which is generated by $G=\langle a, b\rangle$ and an involution $c$, conjugation by which induces the automorphism $\alpha$ on $G$. Also we can let $\Delta^{\prime}$ be an NEC group with signature $(0 ;+;[2],\{(2,3)\})$ and having canonical generators $x_{1}^{\prime}$, $c_{1}^{\prime}, c_{2}^{\prime}, c_{3}^{\prime}$, and then define an epimorphism $\theta^{\prime}: \Delta^{\prime} \rightarrow G^{\prime}=G \rtimes_{\alpha} \mathbb{Z}_{2}$ by setting

$$
\theta^{\prime}\left(x_{1}^{\prime}\right)=a c, \quad \theta^{\prime}\left(c_{1}^{\prime}\right)=c, \quad \theta^{\prime}\left(c_{2}^{\prime}\right)=c b, \quad \text { and } \quad \theta^{\prime}\left(c_{3}^{\prime}\right)=a^{2} c .
$$

Conversely, if such an extension $\theta^{\prime}: \Delta^{\prime} \rightarrow G^{\prime}$ of $\theta$ exists, then by [16] and [5], $\Delta^{\prime}$ must have signature $(0 ;+;[2],\{(2,3)\})$ with canonical generators $x_{1}^{\prime}, c_{1}^{\prime}, c_{2}^{\prime}, c_{3}^{\prime}$, and without loss of generality the embedding of $\Delta$ in $\Delta^{\prime}$ is given by

$$
d_{1} \mapsto x_{1}^{\prime} c_{1}^{\prime}, \quad x_{1} \mapsto c_{1}^{\prime} c_{2}^{\prime}, \quad x_{2} \mapsto c_{2}^{\prime} c_{3}^{\prime} ;
$$

hence if $c$ is the involution $\theta^{\prime}\left(c_{1}^{\prime}\right)$, then

$$
c a c=\theta^{\prime}\left(c_{1}^{\prime} d_{1} c_{1}^{\prime}\right)=\theta^{\prime}\left(c_{1}^{\prime} x_{1}^{\prime}\right)=\theta\left(d_{1}\right)^{-1}=a^{-1}
$$

and

$$
c b c=\theta^{\prime}\left(c_{1}^{\prime} x_{1} c_{1}^{\prime}\right)=\theta^{\prime}\left(c_{2}^{\prime} c_{1}^{\prime}\right)=\theta\left(x_{1}\right)^{-1}=b^{-1},
$$

so conjugation by $c$ gives the required automorphism.

The last proposition and a theorem to follow provide a link with the theory of 3 -valent regular maps.

Definition 5.3. An orientably-regular map $M$ is a 2-cell embedding of a connected graph into an orientable surface, such that the group $\mathrm{Aut}^{+}(M)$ of all orientationpreserving automorphisms of the surface that preserve the embedding has a single orbit on the arcs (directed edges) of the graph. The map is called reflexible if there exist orientation-reversing automorphisms that preserve the embedding, and otherwise it is said to be chiral.

More details may be found in [17] and also in [11, where all orientably-regular maps of genus 2 to 15 were determined. If $M$ is an orientably-regular map of type $\{m, n\}$ (with vertices of valence $m$ and faces of size $n$ ), then $\mathrm{Aut}^{+}(M)$ is generated by two elements $R$ and $S$ satisfying $R^{m}=S^{n}=(R S)^{2}=1$, and $M$ is reflexible if and only if there is an automorphism $\tau$ of $G=\langle R, S\rangle$ such that $\tau(R)=R^{-1}$ and $\tau(S)=S^{-1}$ (or equivalently, an automorphism inverting any one of the pairs $(R, S),(R, R S)$ or $(S, R S))$.

Theorem 5.4. Let $M$ be a chiral regular map of type $\{3, n\}$, where $n$ is odd. If $\operatorname{Aut}(M)=G$, then there exists a pseudo-real Riemann surface $X$ with automorphism group of maximal order and isomorphic to $G \times \mathbb{Z}_{4}$.

Proof. Let $\Delta$ be the $(2,3, n)$ triangle group, with signature $(0 ;[2,3, n])$ and canonical presentation

$$
\left\langle x_{1}, x_{2}, x_{3}: x_{1} x_{2} x_{3}=1, x_{1}^{2}=x_{2}^{3}=x_{3}^{n}=1\right\rangle .
$$

Also let $\theta: \Delta \rightarrow G$ be the epimorphism that corresponds to a chiral regular map of type $\{3, n\}$, taking (say) $x_{1}, x_{2}$ and $x_{3}$ to the automorphisms $R S, R$ and $S$ of $M$, so that $\operatorname{ker} \theta$ is a surface group, and let $b$ be an element of $G$ such that $b^{2}=\theta\left(x_{3}\right)$, which is known to exist because $n$ is odd.

Now let $\Lambda$ be an NEC group with signature $(1 ;-;[2,3])$ and canonical presentation

$$
\left\langle d, y_{1}, y_{2}: y_{1} y_{2} d^{2}=1, y_{1}^{2}=y_{2}^{3}=1\right\rangle,
$$


and define an epimorphism $\omega: \Lambda \rightarrow G \times \mathbb{Z}_{4}=G \times\left\langle a: a^{4}=1\right\rangle$ by setting

$$
\omega(d)=(b, a), \quad \omega\left(y_{1}\right)=\left(\theta\left(x_{1}\right), a^{2}\right) \quad \text { and } \quad \omega\left(y_{2}\right)=\left(\theta\left(x_{2}\right), 1\right) .
$$

Then $\Gamma=\operatorname{ker} \omega$ is a surface group, and since the image of the index 2 subgroup $\left\langle x_{1}, x_{2}, d x_{1} d, d x_{2} d\right\rangle$ of $\Lambda$ is the subgroup $G \times\left\langle a^{2}\right\rangle$ of index 2 in $G \times\langle a\rangle$, the surface $X=\mathbb{D} / \Gamma$ (with automorphism group $\Lambda / \Gamma \cong G \times\langle a\rangle$ ) is orientable; see [5, Theorem 2.1.3 (2)]. Moreover, every element of $G \times\langle a\rangle$ lying outside the orientationpreserving subgroup $G \times\left\langle a^{2}\right\rangle$ is of the form $\left(u, a^{ \pm 1}\right)$ for some $u \in G$, and it follows that every anticonformal automorphism of $X=\mathbb{D} / \Gamma$ has order divisible by 4 . Hence the surface $X$ is pseudo-real. Finally, Theorem 5.1 shows that $\operatorname{Aut}(X)=G$, which has maximal order.

In the following section, we prove that for every integer $k \geq 7$, there exist chiral regular maps of type $\{3, k\}$ on orientable surfaces of infinitely many genera. Using Theorem [5.4, we therefore obtain the following theorem:

Theorem 5.5. There exist pseudo-real surfaces with automorphism group of maximal order, for infinitely many genera. In particular there are infinitely many pseudo-real Riemann surfaces with maximal automorphism group.

\section{Chiral 3-valent Regular maps}

In the previous section we proved that from every chiral regular map of type $\{3, n\}$ for $n$ odd, we can construct a pseudo-real Riemann surface with maximal symmetry. In this section we shall find explicit families of chiral 3-valent regular maps (in Corollary 6.2), which in turn give rise to such pseudo-real Riemann surfaces (by Theorem 5.4).

Theorem 6.1. For every prime $p$ congruent to 1,2 or $4 \bmod 7$, there exists a normal subgroup $K_{p}$ of index $168 p^{3}$ in the ordinary triangle group $\Delta=\Delta(2,3,7)$ such that $\Delta / K_{p}$ is isomorphic to an extension by $P S L(2,7)$ of the 3-generator abelian group $\mathbb{Z}_{p} \times \mathbb{Z}_{p} \times \mathbb{Z}_{p}$ of order $p^{3}$ and exponent $p$. Moreover, the subgroup $K_{p}$ is not normal in the extended triangle group $\Delta^{*}(2,3,7)$, so $\Delta / K_{p}$ has no automorphism that inverts the images of the two generators $x$ and $y$ of $\Delta=\Delta(2,3,7)$.

Note: Here $\Delta$ has signature $(0 ;+;[2,3,7] ;\{-\})$, while the extended triangle group $\Delta^{*}$ has signature $(1 ;+;[-] ;\{(2,3,7)\})$, with $\left(\Delta^{*}\right)^{+} \cong \Delta$.

Corollary 6.2. There exist chiral regular maps of type $\{3,7\}$ on orientable surfaces of infinitely many genera.

Proof of Theorem. Most of this follows from observations made by Leech in 14 and pursued by Cohen in 7, and explained also in [9, 10. First, the extended triangle group $\Delta^{*}=\Delta^{*}(2,3,7)$ has a normal subgroup $N$ of index 336, generated by $a_{0}=[y, x]^{4}$ and its conjugates, such that $\Delta^{*} / N$ is isomorphic to $\operatorname{PGL}(2,7)$. By observations made by Leech 14, this normal subgroup $N$ has a nice presentation in terms of six generators and a single relation (in which each of the generators appears twice, with exponents \pm 1 ).

Now for each prime $p$ as given in the statement of the theorem, let $N_{p}$ denote the normal subgroup of $\Delta^{*}$ generated by the derived subgroup $N^{\prime}=[N, N]$ of $N$ and the set $N^{p}$ of all $p$ th powers of elements of $N$. Then $N_{p}=N^{\prime} N^{p}$ has index $p^{6}$ in $N$, and is normal in $\Delta^{*}$, with quotient $N / N_{p}$ elementary abelian of order $p^{6}$. Moreover, by observations made by Cohen [7] about the action of $\operatorname{PSL}(2,7)$ 
on $N / N_{p}$ induced by conjugation of $N$ by elements of $\Delta=\Delta(2,3,7)$, there exist intermediate subgroups $L_{1}$ and $L_{2}$ of $N$ containing $N_{p}$, such that each $L_{i}$ is normal in $\Delta$, and $N=L_{1} L_{2}$ with $L_{1} \cap L_{2}=N_{p}$, and with $N / L_{i}$ elementary abelian of order $p^{3}$ for $i \in\{1,2\}$. On the other hand, $L_{1}$ and $L_{2}$ are not normal in the extended triangle group $\Delta^{*}=\Delta^{*}(2,3,7)$; indeed every element of $\Delta^{*} \backslash \Delta$ conjugates $L_{1}$ to $L_{2}$ and vice versa. Hence we can take $K_{p}=L_{1}$ or $L_{2}$, to give the required result.

Theorem 6.3. For every integer $k \geq 7$, all but finitely many of the alternating groups $A_{n}$ can be generated by two elements $x$ and $y$ such that $x, y$ and $x y$ have orders 2, 3 and $k$ respectively, and such that there exists no automorphism of $\langle x, y\rangle=A_{n}$ taking $x$ and $y$ to $x^{-1}$ and $y^{-1}$, respectively.

Corollary 6.4. For each integer $k \geq 7$, there exist chiral regular maps of type $\{3, k\}$ on orientable surfaces of infinitely many genera.

Proof of Theorem. In all cases our argument relies heavily on a construction used by the second author in $\left[8\right.$ to prove that (for every $k \geq 7$ ) all but finitely many $A_{n}$ are homomorphic images of the extended triangle group

$$
\Delta^{*}(2,3, k)=\left\langle x, y, t \mid x^{2}=y^{3}=(x y)^{k}=t^{2}=(x t)^{2}=(y t)^{2}=1\right\rangle,
$$

a group with signature $(1 ;+;[-] ;\{(2,3, k)\})$. We refer the reader to 8 ] for important details. In that construction, permutation representations of $\Delta^{*}(2,3, k)$ are depicted by Schreier coset diagrams, and specially chosen examples of such diagrams are linked together to form representations of arbitrarily large degree $n$, in a way that makes the resulting permutations generate $A_{n}$ or $S_{n}$. We will amend that construction by adding one more small diagram that depicts a permutation representation of the ordinary triangle group $\Delta=\left(\Delta^{*}\right)^{+}$, but does not depict one of the extended triangle group $\Delta^{*}(2,3, k)$ itself. Note that $\Delta=\Delta(2,3, k)$ is the index 2 subgroup of $\Delta^{*}(2,3, k)$ generated by $x$ and $y$.

We do this first for the case $k=7$, and then explain in less detail how the theorem can be proved for larger $k$ using the same method.

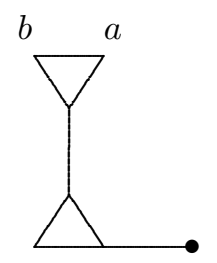

Figure 1. Additional coset diagram $R(7,0)$ with 7 vertices

When $k=7$, consider the permutation representation of $\Delta(2,3,7)$ on 7 points given by the diagram $R(7,0)$ in Figure 1, Like the diagrams $S(7,0), T(7,0), U(7,0)$ and $V(7,0)$ in $\left[8\right.$, this has a (1)-handle $[a, b]_{1}$, consisting of two points $a$ and $b$ such that $x$ fixes both $a$ and $b$, and $y$ takes $a$ to $b$. Note that the point $a$ is fixed by the commutator $x y x y^{-1}$, while $b$ lies in a 2-cycle of $x y x y^{-1}$, and the other four points lie in a 4-cycle. Similarly, if $\left[a^{\prime}, b^{\prime}\right]_{1}$ is a (1)-handle of the diagram $S(7,0)$, then $a^{\prime}$ is fixed by $x y x y^{-1}$, while $b^{\prime}$ lies in a 13 -cycle of $x y x y^{-1}$, consisting of the 13 points of the cycle of $x y t$ in the representation of $\Delta(2,3,7)$ that it depicts. Indeed it follows from the relations for the extended triangle group $\Delta^{*}(2,3,7)$ that 
$(x y t)^{2}=x y t x y t=x y x t y t=x y x y^{-1}$, and hence the cycle structure of $x y x y^{-1}$ can be derived easily from that of $x y t$ on the points of the diagram $S(7,0)$.

Next suppose that a single copy of the diagram $R(7,0)$ is linked together with a single copy of the diagram $S(7,0)$, by adding the transpositions $\left(a, a^{\prime}\right)$ and $\left(b, b^{\prime}\right)$ to the permutation induced by $x$ (while not altering the permutation induced by $y$ ). Then the resulting diagram is easily seen to be a coset diagram for the ordinary triangle group $\Delta(2,3,7)$, by the same argument as in 8 . Also in the corresponding permutation representation, the two points $a$ and $a^{\prime}$ are still both fixed by $x y x y^{-1}$, while the cycles containing $b$ and $b^{\prime}$ and the other four points of the diagram $R(7,0)$ are joined together to form a new cycle of $x y x y^{-1}$, of length 19. (This is easily verified, either by writing out the permutations, or by chasing points around the combined diagrams.)

The construction in [8 explains how a transitive permutation representation of $\Delta^{*}(2,3,7)$ on $n=42 f+71 g+36$ points (when $f>g \geq 0$ ) can be formed by linking together $f$ copies of diagram $S(7,0)$ and then adjoining $g$ copies of $T(7,0)$ and a single copy of $U(7,0)$, by composition of (1)-handles. In the resulting representation, the element $x y t$ has cycle structure

$$
1^{f+1-g} 2^{f+g} 5^{1} 6^{g} 8^{1} 11^{1} 13^{f+1-g} 15^{g} 20^{g} 24^{1} 26^{f-1} 42^{g},
$$

so the commutator $x y x y^{-1}$ has cycle structure

$$
1^{3 f+1} 3^{2 g} 4^{2} 5^{1} 11^{1} 13^{3 f-1-g} 10^{2 g} 12^{2} 15^{g} 21^{2 g} .
$$

The unique 11-cycle here comes from the single copy of $U(7,0)$, and this can be used (with the help of Jordan's theorem from [19]) to prove that the permutations induced by $x, y$ and $t$ generate $S_{n}$, while those induced by $x$ and $y$ generate $A_{n}$.

Now suppose that a single copy of the diagram $R(7,0)$ is linked to one of the copies of $S(7,0)$ still having a free (1)-handle in this representation. Then we have a new transitive permutation representation of $\Delta(2,3,7)$ on $n+7$ points, in which $x y x y^{-1}$ has cycle structure

$$
1^{3 f+2} 3^{2 g} 4^{2} 5^{1} 11^{1} 13^{3 f-2-g} 10^{2 g} 12^{2} 15^{g} 19^{1} 21^{2 g} .
$$

Again the unique 11-cycle here comes from the single copy of $U(7,0)$ and can be used to prove that the permutations induced by $x$ and $y$ generate $A_{n+7}$. An important difference this time, however, is that because the point fixed by $y$ in the single copy of $R(7,0)$ is the only point fixed by $y$ that lies close to a fixed point of $x y x y^{-1}$ or $x y^{-1} x y$ in the resulting coset diagram (on $n+7$ points), this diagram has no axis of reflectional symmetry. Thus we have a homomorphism from $\Delta(2,3,7)$ to $A_{n+7}$ that does not extend to a representation of the extended triangle group $\Delta^{*}(2,3,7)$, as claimed.

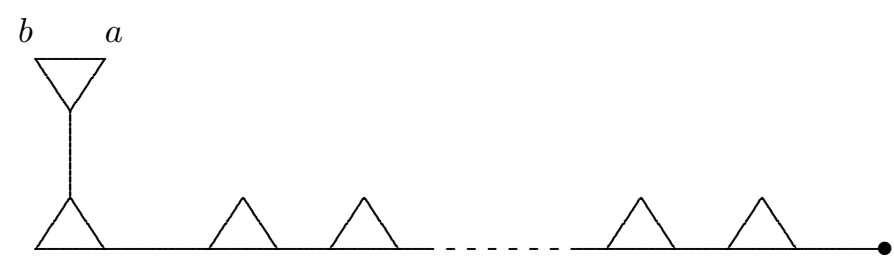

FIgURE 2. Additional coset diagram $R(7, d)$ with $7+6 d$ vertices 
When $k=7+6 d$ for some positive integer $d$, we can apply the same construction using $S(7, d), T(7, d)$ and $U(7, d)$ from $[8$, and add a single copy of the new coset diagram $R(7, d)$ for $\Delta(2,3,7+6 d)$ on $7+6 d$ points given in Figure 2 ,

In the permutation representation of $\Delta(2,3,7+6 d)$ depicted by $R(7, d)$, the commutator $x y x y^{-1}$ fixes the point $a$, and has two 2-cycles, two 4-cycles, and $2(d-1) 3$-cycles. Linking a single copy of $R(7, d)$ to a copy of $S(7, d)$ by their free (1)-handles gives rise to a new permutation representation of $\Delta(2,3,7+6 d)$ in which one of the 2-cycles and one of the 4-cycles from $R(7, d)$ are combined together with two of the cycles from $S(7, d)$ to form a 7 -cycle and a 10-cycle when $d=1$, or a 6 -cycle and an 8-cycle when $d \geq 2$.

Thus again we can form transitive permutation representations of $\Delta(2,3,7+6 d)$ of arbitrarily large degree, use the unique 11-cycle from the single copy of $U(7, d)$ to prove that the resulting permutations generate an alternating group, and use the single copy of $R(7, d)$ to eliminate the possibility of a reflectional symmetry.

The proof for other cases (with $k$ in different congruence classes mod 6) is analogous to the above, using the additional diagrams given in Figures 3 to 7 below.

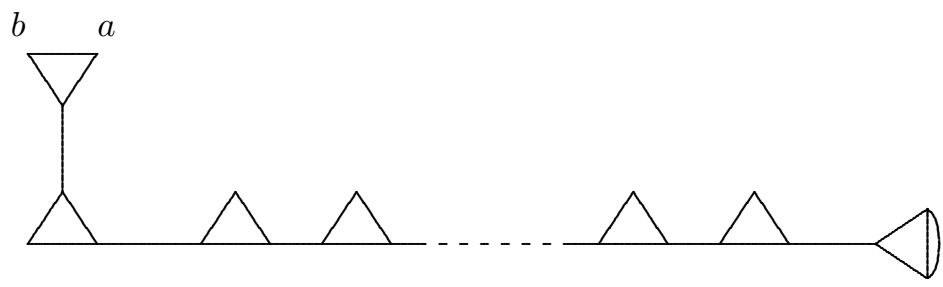

Figure 3. Additional coset diagram $R(8, d)$ with $8+6 d$ vertices

For the case $k=8+6 d$, joining a single copy of diagram $R(8, d)$ replaces cycles of $x y x y^{-1}$ by one cycle of length 15 if $d=0$, or cycles of length 7 and 9 if $d=1$, or cycles of length 6 and 8 if $d \geq 2$, leaving a unique 11-cycle from the single copy of diagram $U(8, d)$ for application of Jordan's theorem.

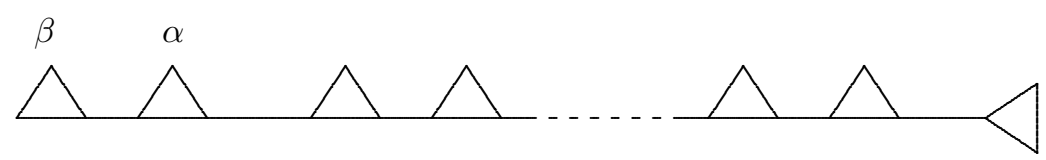

Figure 4. Additional coset diagram $R(9, d)$ with $9+6 d$ vertices

For the case $k=9+6 d$, diagrams are composed using (2)-handles $[\alpha, \beta]_{2}$, consisting of fixed points $\alpha$ and $\beta$ such that $y^{2}$ takes $\alpha$ to $\beta$. Joining a single copy of diagram $R(9, d)$ replaces cycles of $x y x y^{-1}$ by cycles of length 12 and 14 if $d=0$, or cycles of length $3,5,9$ and 10 if $d=1$, or cycles of length $3,5,6$ and 7 if $d \geq 2$, leaving a unique 13-cycle from the single copy of diagram $U(9, d)$ for application of Jordan's theorem.

For the case $k=10+6 d$, joining a single copy of diagram $R(10, d)$ replaces cycles of $x y x y^{-1}$ by cycles of length $6,7,7$ and 10 if $d=0$, or cycles of length $5,5,6$ and 7 if $d=1$, or cycles of length 4, 5, 6 and 6 if $d \geq 2$, leaving a unique 13-cycle from the single copy of diagram $U(10, d)$ for application of Jordan's theorem. 


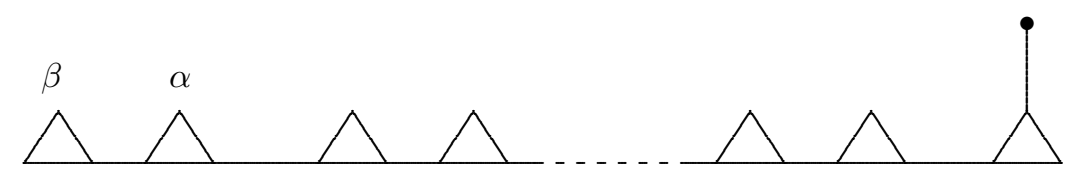

Figure 5. Additional coset diagram $R(10, d)$ with $10+6 d$ vertices

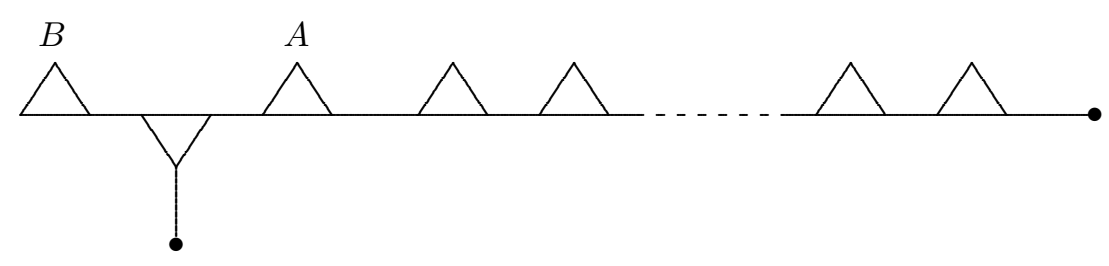

Figure 6 . Additional coset diagram $R(11, d)$ with $11+6 d$ vertices

For the case $k=11+6 d$, joining a single copy of diagram $R(11, d)$ replaces cycles of $x y x y^{-1}$ by cycles of length 9 and 19 if $d=0$, or cycles of length 6,8 and 9 if $d \geq 1$, leaving a unique 11-cycle from adjoining the single copy of diagram $U(11, d)$ for application of Jordan's theorem.

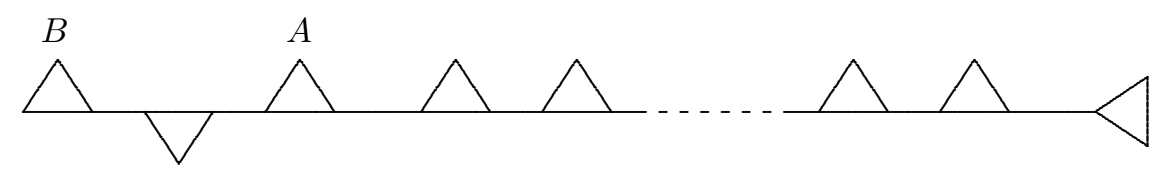

Figure 7. Additional coset diagram $R(12, d)$ with $12+6 d$ vertices

For the case $k=12+6 d$, joining a single copy of diagram $R(12, d)$ replaces cycles of $x y x y^{-1}$ by cycles of length 9 and 10 if $d=0$, or cycles of length 5,7 and 9 if $d=1$, or cycles of length 4,6 and 9 if $d \geq 2$, leaving a unique 13-cycle from adjoining the single copy of diagram $U(12, d)$ for application of Jordan's theorem.

\section{REFERENCES}

1. R.D.M. Accola, Strongly branched coverings of closed Riemann surfaces, Proc. Amer. Math. Soc. 26 (1970), 315-322. MR0262485(41:7091)

2. E. Bujalance, M. Conder, On cyclic groups of automorphisms of Riemann surfaces, J. London Math. Soc. (2) 59 (1999), no. 2, 573-584. MR1709666 (2000g:20098)

3. E. Bujalance, F.J. Cirre, M. Conder, On extendability of group actions on compact Riemann surfaces, Trans. Amer. Math. Soc. 355 (2003), no. 4, 1537-1557. MR1946404 (2003k:20079)

4. E. Bujalance, A.F. Costa, A. Fernandez, Uniformization of Klein surfaces by maximal NEC groups. Travaux de la Conférence Internationale d'Analyse Complexe et du 7e Séminaire Roumano-Finlandais (1993), Rev. Roumaine Math. Pures Appl. 40 (1995), no. 1, 39-54. MR.1406119 (97g:30043)

5. E. Bujalance, J.J. Etayo, J.M. Gamboa, G. Gromadzki, Automorphism groups of compact bordered Klein surfaces. A combinatorial approach. Lecture Notes in Mathematics, 1439. Springer-Verlag, Berlin, 1990. xiv+201 pp. MR.1075411 (92a:14018) 
6. S.A. Broughton, Classifying finite group actions on surfaces of low genus, J. Pure Appl. Algebra 69 (1991), no. 3, 233-270. MR1090743 (92b:57021)

7. J.M. Cohen, On Hurwitz extensions by $P S L_{2}(7)$, Math. Proc. Cambridge Philos. Soc. 86 (1979), 395-400. MR.542684 (80j:30069)

8. M.D.E. Conder, More on generators for alternating and symmetric groups, Quart. J. Math. Oxford (2) 32 (1981), 137-163. MR615190 (82e:20001)

9. M.D.E. Conder, The genus of compact Riemann surfaces with maximal automorphism group, J. Algebra 108 (1987), 204-247. MR887205 (88f:20063)

10. M.D.E. Conder, Maximal automorphism groups of symmetric Riemann surfaces with small genus, J. Algebra 114 (1988), 16-28. MR931896 (89c:20049)

11. M.D.E. Conder, P. Dobcsányi, Determination of all regular maps of small genus, J. Combin. Theory Ser. B 81 (2001), 224-242. MR.1814906 (2002f:05088)

12. C.J. Earle, On the moduli of closed Riemann surfaces with symmetries, in Advances in the theory of Riemann surfaces (Proc. Conf., Stony Brook, N.Y., 1969), pp. 119-130. Ann. of Math. Studies, No. 66, Princeton Univ. Press, Princeton, N.J., 1971. MR0296282 (45:5343)

13. J.J. Etayo Gordejuela, Nonorientable automorphisms of Riemann surfaces, Arch. Math. (Basel) 45 (1985), no. 4, 374-384. MR810257(87c:20087)

14. J. Leech, Generators for certain normal subgroups of $(2,3,7)$, Proc. Cambridge Philos. Soc. 61 (1965), 321-332. MR0174621(30:4821)

15. S.M. Natanzon, Moduli of Riemann surfaces, real algebraic curves, and their superanalogs. Translated from the 2003 Russian edition by Sergei Lando. Translations of Mathematical Monographs, 225. American Mathematical Society, Providence, RI, 2004. viii+160 pp. MR2075914 (2005d:32020)

16. D. Singerman, Finitely maximal Fuchsian groups, J. London Math. Soc. (2) 6 (1972), 29-38. MR $0322165(48: 529)$

17. D. Singerman, Symmetries of Riemann surfaces with large automorphism group, Math. Ann. 210 (1974), 17-32. MR0361059 (50:13505)

18. D. Singerman, Symmetries and pseudo-symmetries of hyperelliptic surfaces, Glasgow Math. J. (1) 21 (1980), 39-49. MR.558273 (81c:30080)

19. H. Wielandt, Finite Permutation Groups, Academic Press (New York), 1964. MR0183775 $(32: 1252)$

Departamento de Matemáticas Fundamentales, Facultad de Ciencias, Universidad Nacional de Educacion a Distancia, Senda del Rey, 9, 28040 Madrid, Spain

E-mail address: eb@mat.uned.es

Department of Mathematics, University of Auckland, Private Bag 92019, Auckland, NeW ZEALAND

E-mail address: m.conder@auckland.ac.nz

Departamento de Matemáticas Fundamentales, Facultad de Ciencias, Universidad Nacional de Educacion a Distancia, Senda del Rey, 9, 28040 Madrid, Spain

E-mail address: acosta@mat.uned.es 